\title{
Paraneoplastic pemphigus with anti-desmocollin 3 autoantibodies and chronic lymphocytic leukemia
}

\author{
Alicja Adaszewska ${ }^{1}$, Norito Ishii ${ }^{2}$, Jadwiga Dwilewicz-Trojaczek³ ${ }^{3}$ Katarzyna Woźniak ${ }^{1}$, Takashi Hashimoto², \\ Cezary Kowalewski ${ }^{1}$
}

${ }^{1}$ Department of Dermatology and Immunodermatology, Medical University of Warsaw, Warsaw, Poland 2Department of Dermatology, Kurume University School of Medicine, and Kurume University Institute of Cutaneous Cell Biology, Kurume, Japan

${ }^{3}$ Department of Haematology and Oncology, Medical University of Warsaw, Warsaw, Poland

Adv Dermatol Allergol 2018; XXXV (1): 113-115

DOI: https://doi.org/10.5114/ada.2018.73171

Paraneoplastic pemphigus (PNP) is an autoimmune blistering disorder coexisting with certain neoplasms, most commonly non-Hodgkin lymphoma, chronic lymphocytic leukemia (CLL) and Castleman's disease [1]. The clinical presentation of PNP consists of painful mucosal erosions, polymorphic cutaneous lesions and systemic involvement. The PNP is characterized by various autoantibodies directed mainly to desmosomal proteins, particularly various plakin family proteins [2]. Recently the $170 \mathrm{kDa} \alpha_{2}$-macroglobulin-like protein 1 was identified as another PNP antigen [3]. In addition, novel ELISA of mammalian recombinant proteins (RP)s detected autoantibodies to desmocollins 1 (Dsc1) to Dsc3 in 67\% of PNP cases [4]. Herein, we report an unusual case of PNP associated with CLL, mediated by antibodies against Dsc3.

In July 2013, a 50-year-old man was admitted to our department with a 6-week history of painful erosions in the oral cavity (Figure $1 \mathrm{~A}$ ) and significant weight loss (6 kg within 1 month). Direct immunofluorescence (IF) of oral mucosal biopsy and indirect IF of monkey esophagus and human skin showed negative results. The diagnosis of Stevens-Johnson syndrome was made and the patient was treated with prednisolone $40 \mathrm{mg} /$ day and acyclovir $400 \mathrm{mg} /$ day for 1 month with no improvement. After systemic corticosteroids started the patient developed vegetating oral mucosal lesions (Figure 1 B). However, repeated direct IF for oral mucosal lesions remained negative.

Five weeks later the patient presented extensive erosive lesions both in the oral and on the genital mucosa (Figure 1 C). Subsequently, the patient developed disseminated vesicular skin lesions on the trunk and the upper extremities and damaged finger nails (Figure 1D). A blood test performed at that time disclosed leukocytosis of 20,000 U/I with lymphocytosis. The immunopathological studies were repeated. Direct IF of perilesional skin showed IgG and C3 deposits both on keratinocyte cell surfaces and linearly along the epidermal basement membrane zone (BMZ) (Figure $2 \mathrm{~A}$ ). Indirect IF of normal human skin, monkey esophagus and guinea pig disclosed circulating IgG anti-cell surface antibodies. Indirect IF of rat bladder also showed positive reaction with transitional epithelia (Figure 2 B). However, indirect IF of $1 \mathrm{M} \mathrm{NaCl}$-split normal human skin showed negative results. Immunoblot (IB) studies were performed using a battery of antigen sources. mmunoblot of normal human epidermal extract was positive for the $210 \mathrm{kDa}$ envoplakin and $190 \mathrm{kDa}$ periplakin, but negative for the $130 \mathrm{kDa}$ desmoglein 3 (Dsg3) and the $160 \mathrm{kDa}$ Dsg1, BP230 and BP180 (Figure 2 C). There was no positive reaction in IB studies of RPs of both NC16a and C-terminal domains of BP180, concentrated culture supernatant of $\mathrm{HaCaT}$ cells, normal human dermal extract or purified human laminin-332 (Figure 2 C). Commercially available ELISAs (MBL, Nagoy, Japan) showed a positive reaction with Dsg3 (index value 53.67, cut-off < 14) but not with Dsg1. Novel ELISAs using mammalian RPs of Dsc1-3 [4] detected IgG antibodies to Dsc3 (OD 1.17, cut-off < 0.120), but not to Dsc1 or Dsc2. Trepan biopsy led to the diagnosis of CLL. From these findings, the case was diagnosed as PNP associated with CLL. Pulse therapy consisting of intravenous rituximab $\left(375 \mathrm{mg} / \mathrm{m}^{2}\right)$, cyclophosphamide $1400 \mathrm{mg} /$ day and dexamethasone $12 \mathrm{mg} /$ day was initiated. Between administrations of chemotherapy at 1-month intervals, prednisolone $30 \mathrm{mg} /$ day was given. One month after the first pulse of chemotherapy, skin and mucous lesions significantly improved, whereas CLL came into remission after eight pulses of chemotherapy.

The diagnosis of PNP is often challenging since skin manifestations are initially diverse, resembling erythema multiforme, Stevens-Johnson syndrome or

Address for correspondence: Alicja Adaszewska MD, Department of Dermatology and Immunodermatology, Medical University of Warsaw, 82 A Koszykowa St, 05-080 Warsaw, Poland, phone: +48 607248 335, e-mail: alicja.adaszewska@gmail.com Received: 22.02.2017, accepted: 4.03.2017. 

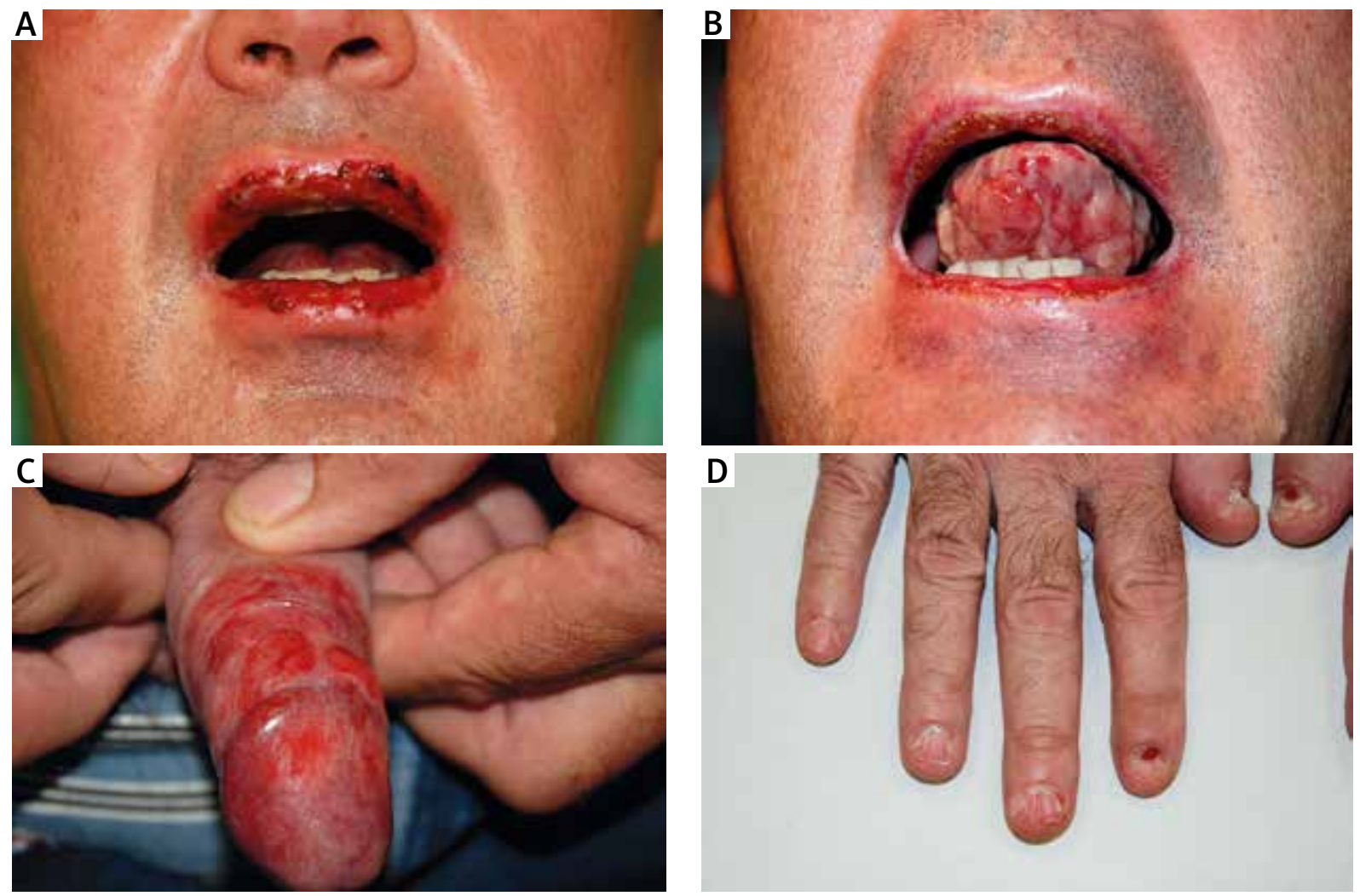

Figure 1. A - Painful erosions on the lips at the onset of the disease. B - Vegetating oral lesions 3 months later. $\mathrm{C}$ - Erosions on the genital mucosa. D - Damaged finger nails

lichenoid disorders. Deposition of IgG and C3 both to the epithelial cell surfaces and along the BMZ in direct IF of skin or mucosal biopsies is characteristic for PNP

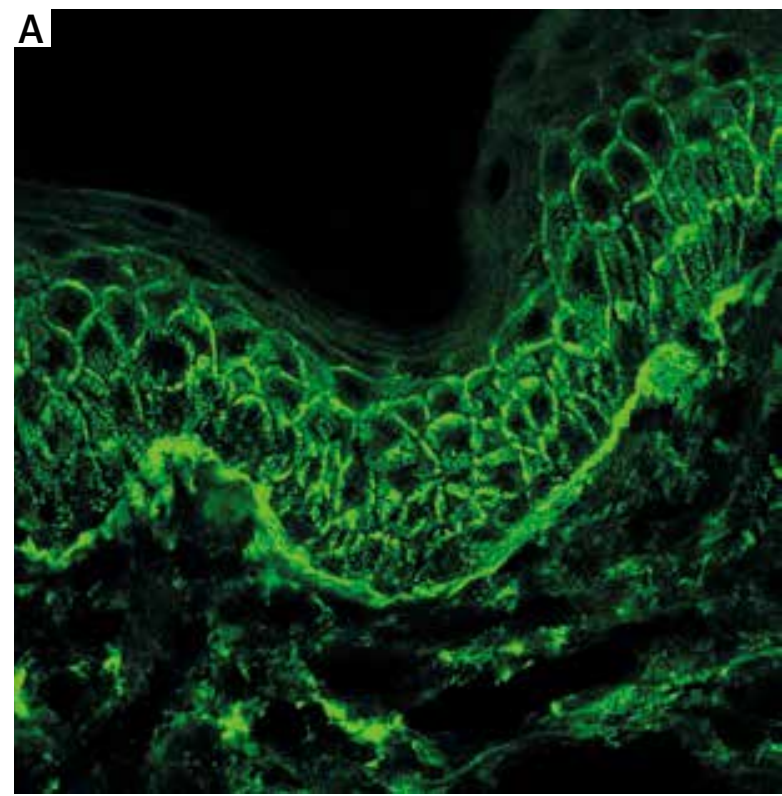

[5]. Herein, we report a case of PNP associated with CLL, which initially presented severe oral vegetating erosions with repeated negative results of direct and indirect IF.

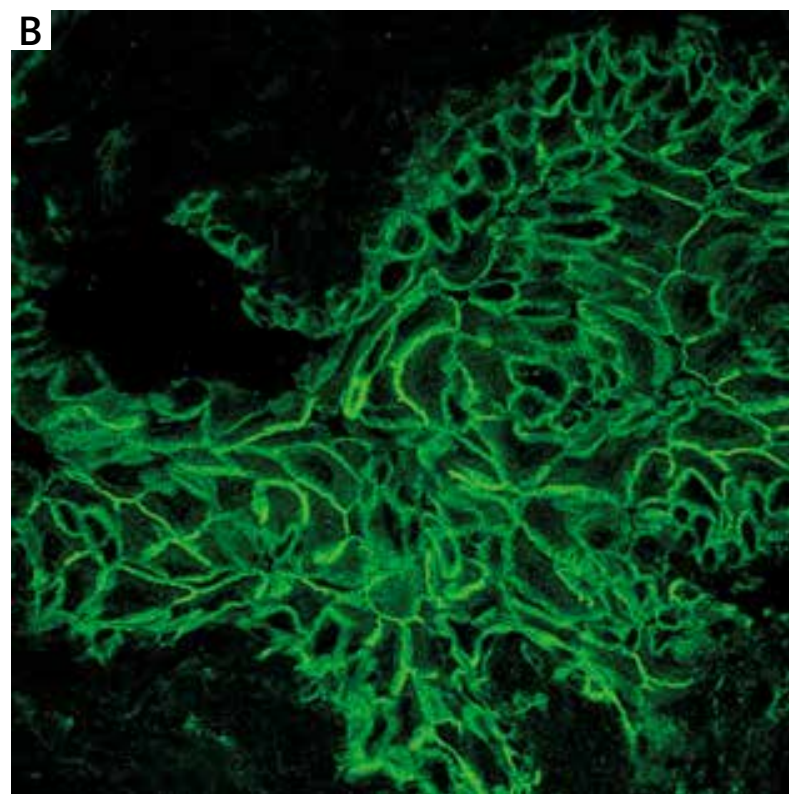

Figure 2. A - Direct IF showing IgG deposition on the cell surfaces and linearly along the BMZ. B - Indirect IF of a rat bladder showing reactivity with transitional epithelium at a titer of $1: 40$. 
C

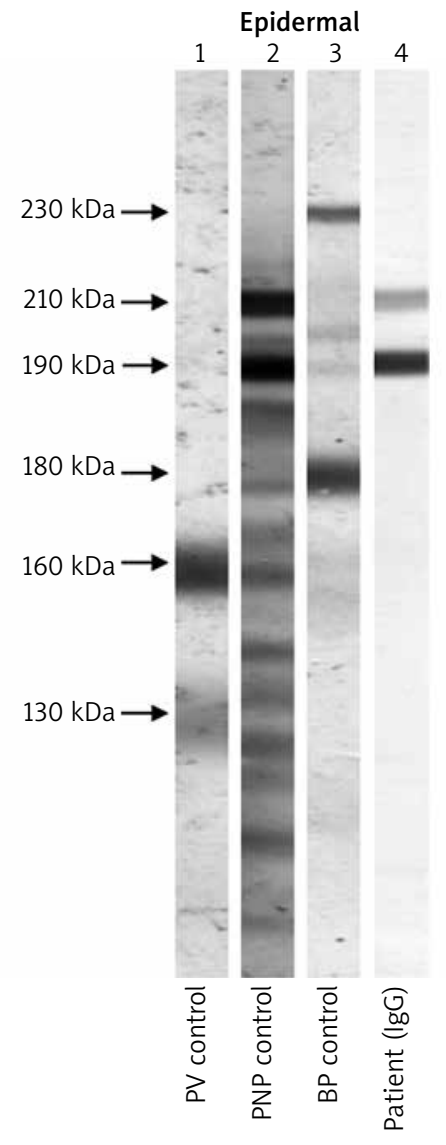

BP180-NC16a

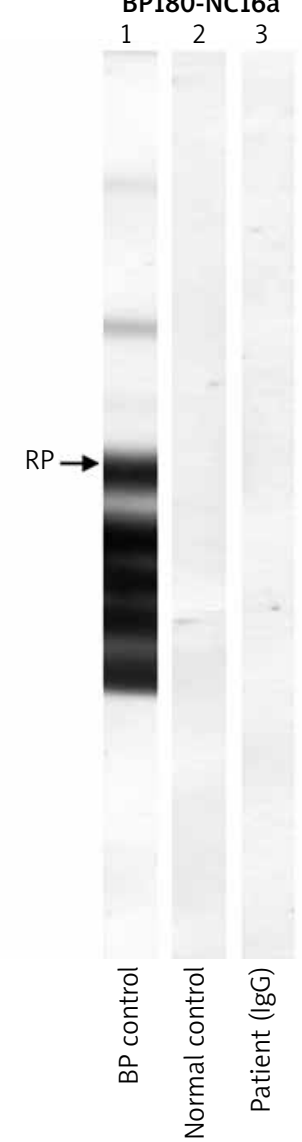

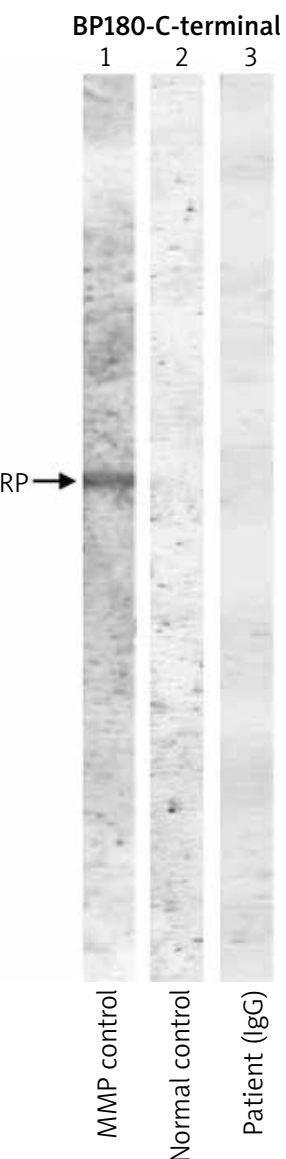

Figure 2. C - Immunoblot studies of a battery of antigen sources showing positive reactivity exclusively with periplakin and envoplakin in normal human epidermal extract

It is likely that Stevens-Johnson syndrome preceded in this case, as reported in patients with other autoimmune bullous diseases [6]. However, it may also be possible that, at the initial phase, our patient produced antibodies exclusively against Dsc3, which were difficult to be detected by routine direct IF, indirect IF and IB studies. Later, anti-Dsc3 antibodies were detected by novel ELISAs of Dsc1-3 RPs. The Dsc ELISA gave a new insight into the pathogenesis of PNP, since $67 \%$ of PNP patients showed antibodies against Dsc1-3 [4]. In addition, anti-Dsc3 antibodies have been reported to be responsible for oral lesions, particularly in cases without anti-Dsg3 antibodies [7].

In the past, immunoprecipitation was used for the diagnosis of PNP [1]. However, due to radioisotope use limitations, a combination of IB and indirect IF on a rat bladder is suggested to be equally sensitive for the diagnosis of PNP [5]. This diagnostic method was performed and proved useful in this study. Since anti-Dsc antibodies are present in the majority of PNP patients and may precede the production of other anti-desmosomal antibodies, novel ELISAs of Dsc1-3 should be added to the panel of laboratory tests for rapid diagnosis of PNP.

\section{Conflict of interest}

The authors declare no conflict of interest.

\section{References}

1. Anhalt GJ, Kim SC, Stanley JR, et al. Paraneoplastic pemphigus. An autoimmune mucocutaneous disease associated with neoplasia. N Engl J Med 1990; 323: 1729-35.

2. Schepens I, Jaunin F, Begre $\mathrm{N}$ et al. The protease inhibitor alpha-2-macroglobulin-like-1 is the p170 antigen recognized by paraneoplastic pemphigus autoantibodies in human. PLoS One 2010; 5: e12250.

3. Hashimoto T. Production of numerous autoantibodies in paraneoplastic pemphigus. Br J Dermatol 2015; 172: 849-50.

4. Ishii N, Teye K, Fukuda S, et al. Anti-desmocollin autoantibodies in non-classical pemphigus. Br J Dermatol 2015; 173 : 59-68.

5. Poot AM, Diercks GF, Kramer D, et. al. Laboratory diagnosis of paraneoplastic pemphigus. Br J Dermatol 2013; 169: 1016-24.

6. Wozniak K, Kowalewski C, Hashimoto T, et al. Penicillin-induced anti-p200 pemphigoid: an unusual morphology. Acta Derm Venereol 2006; 86: 443-6.

7. Mao X, Nagler AR, Farber SA, et al. Autoimmunity to desmocollin 3 in pemphigus vulgaris. Am J Pathol 2010; 177: 2724-30. 